\title{
Practical Experience of Endoscope Reprocessing and Working- Platform Disinfection in COVID-19 Patients: A Report from Guangdong China during the Pandemic
}

\author{
Xianqi Lin, ${ }^{1}$ Zhenyi Zhang, ${ }^{1}$ Minzhao Gao ${ }^{1},{ }^{1}$ Zhenling Zhang, ${ }^{1}$ Zhidong Lin, ${ }^{2}$ \\ Siwen Huang, ${ }^{1}$ Jiangnan Ren, ${ }^{1}$ Meijuan Luo, ${ }^{1}$ Xiaoqing Xu, ${ }^{1}$ Guilan Chen, ${ }^{1}$ Hongjun Chen, ${ }^{1}$ \\ Renxu Lai, ${ }^{1}$ Xiaochun $\mathrm{Wu}^{2}{ }^{2}$ Lihua Zhang $\mathbb{D}^{1},{ }^{1}$ and Xiaofeng $\mathrm{Li} \mathbb{D}^{1}$ \\ ${ }^{1}$ Department of Gastroenterology, The Fifth Affiliated Hospital, Sun Yat-sen University, Zhuhai, 519000 Guangdong, China \\ ${ }^{2}$ Department of Hospital Infection Management, The Fifth Affiliated Hospital, Sun Yat-sen University, Zhuhai, \\ 519000 Guangdong, China \\ Correspondence should be addressed to Lihua Zhang; 18825629232@163.com and Xiaofeng Li; lixiaofeng@mail.sysu.edu.cn
}

Received 22 July 2020; Revised 24 November 2020; Accepted 21 December 2020; Published 31 December 2020

Academic Editor: Suresh Venkateswaran

Copyright (C) 2020 Xianqi Lin et al. This is an open access article distributed under the Creative Commons Attribution License, which permits unrestricted use, distribution, and reproduction in any medium, provided the original work is properly cited.

Background. No consensus exists regarding which procedures should be performed to disinfect endoscopes and working platforms after COVID-19 patients have undergone endoscopy. Methods. We analyzed the disinfection quality of endoscopes and working platforms after 11 COVID-19 patients had undergone endoscopy. Conclusions. For endoscopic preprocessing at the bedside, a key disinfection step is using a multienzyme stock solution. The nucleic acid tests for endoscopists, washers, endoscopes, and working platforms were all negative. Based on our experience with the 11 COVID-19 patients who had undergone endoscopy, we provide an endoscopic reprocessing method for the bedside endoscopic diagnosis and treatment of COVID-19 patients for reference.

\section{Introduction}

Severe acute respiratory syndrome coronavirus 2 (SARS$\mathrm{CoV}-2$ ) produces the disease called coronavirus disease 2019 (COVID-19), which has spread worldwide as a transmissible respiratory disease. The most common symptoms of COVID-19 at the onset of illness are fever, cough, fatigue, myalgia, dyspnea, and GI symptoms [1-5]. The virus can be transmitted by contact, droplets, and aerosols and has been isolated from gastrointestinal secretions and feces [6-8]. Therefore, to avoid the spread of the virus, the cleaning and disinfection of endoscopes and working platforms are essential.

Our hospital, The Fifth Affiliated Hospital, Sun Yat-sen University, is a designated health care provider for COVID19 in Zhuhai, where 103 cases of COVID-19 were treated. From January 17 to February 15, 2020, 11 patients had undergone bedside endoscopy. We focused on infection control and occupational protection against COVID-19 and summarized our experience with 11 COVID-19 patients who had undergone gastrointestinal endoscopy.

\section{Methods}

2.1. Health Care Professional Protection. According to the three-level protection requirements [9], in the buffer area, before entering the negative pressure ward [10], endoscopists and nurses should dress in specialized personal protection equipment (PPE), including medical protective clothing (disposable), impermeable isolation gowns, medical protective caps, waterproof boot covers, medical shoe covers, N95 medical protective masks, full-face respiratory protectors or positive pressure headgear, and double-layered gloves. Endoscope reprocessing operators should wear 
medical protective clothing (disposable), impermeable isolation gowns, medical protective caps, waterproof boot covers, medical shoe covers, N95 medical protective masks, protective face shields, and double-layered gloves in the buffer area before entering the endoscope reprocessing area. The health care professionals should remove their PPE in the buffer area before returning to clean working areas.

\subsection{Endoscope Reprocessing}

2.2.1. Bedside Preprocessing. According to WS507-2016 [11], after endoscopy of the COVID-19 patients, bedside preprocessing of the endoscope was performed. After the completion by the endoscopists, the preprocessing operator immediately spirally scrubbed the outer surface of the endoscope operation part and outer surface of the insertion part with gauze containing multienzyme stock solution to avoid possible hazards of saliva adhering to the endoscope surface. The head end of the endoscope was immersed in the multienzyme stock solution, with repeated injection of gas/water until the tube was free from dirt under visual inspection. Next, $200 \mathrm{ml}$ of the multienzyme stock solution was drawn into and maintained in the endoscope lumen for 2 minutes. To prevent protein solidification and crystallization caused by contact of the mucus on the endoscope lumen with the disinfectant, reduce the virus residue, and prevent the blocking of the endoscope lumen, we increased the concentration of the multienzyme and used the multienzyme stock solution directly. After removing the endoscope, the waterproof cap with the endoscope water injection bottle placed in a double-deck yellow medical bag (gooseneck type) was installed and labeled with the patient information and the word "COVID-19." The medical bag was then sealed and transported through a special channel to the designated endoscope reprocessing room.

\subsubsection{Endoscopic Cleaning. Endoscope reprocessing opera-} tors performed three-level protection as required and wore a face shield to prevent the generation of aerosol during the cleaning process. The endoscope was completely immersed in the tank of multienzyme solution, the endoscope body was repeatedly scrubbed with a gauze block, and the head end and operation part were mainly scrubbed. Next, the endoscope biopsy pipeline was washed with a pressure water gun. The opening valve, water supply and air supply button, and suction button were cleaned and soaked in the multienzyme cleaning solution. A disposable endoscope cleaning brush was used to scrub the endoscopic biopsy pipe and suction pipe. When brushing, the brush head at both ends was visible and the dirt was cleaned on the brush head. Brushing was performed repeatedly until no debris and tissues were visible to the naked eye (the brush was rotated when entering and withdrawing). The brush head was washed again, rinsed with a pressure water gun, and blow dried with a pressure air gun (the level was not higher than the groove surface to minimize the generation of aerosols). A dedicated button for endoscope cleaning was installed, the injection port in the automatic perfusion device was connected to the endoscope perfusion connection tube, and the "start" button of the full-channel perfusion device was touched to fully brush and clean each lumen of the endoscope.

2.2.3. Endoscopic Rinse. The cleaned endoscope, its components, and accessories were placed in the rinsing tank with flowing water. The full-tube perfusion device was turned on for full perfusion cleaning, and each pipe of the endoscope was thoroughly rinsed for at least 10 seconds, followed by rinsing the outer surface, parts, and accessories of the endoscope with flowing water. A pressure air gun was used to remove the moisture in the pipe and blow dry. Finally, the outer surface, parts, and accessories of the endoscope were dried.

2.2.4. Endoscope Disinfection. The endoscope was completely immersed in the $0.55 \%$ orthophthalaldehyde (OPA) disinfectant for 5 minutes, and the endoscope pipe was filled with disinfectant using a fully automatic perfusion device or syringe, which disinfects for the prescribed time.

2.2.5. Final Rinse. Hands were washed with flowing water, and double gloves were replaced. Next, the perfusion connector was removed, and then, the endoscope was removed from the disinfection tank and placed in the final rinse tank. The full-tube perfusion device (or pressure air gun, syringe) was connected to inflate various pipelines and discharge the disinfectant solution in the pipeline. Additionally, the full-tube perfusion device (or pressure gun, syringe) repeatedly rinsed each pipe of the endoscope with pure water for at least 2 minutes and fully rinsed the outer surface of the endoscope and accessories.

2.2.6. Drying. The endoscope subjected to terminal rinse was placed on the drying table where disposable sterile treatment towels were placed. The full-tube perfusion device (or pressure air gun, syringe) was turned on, and all the tubes were flushed with $75 \%$ ethanol until the insertion end of the endoscope was full, and then, the endoscope lumen was filled with $75 \%$ ethanol and maintained for at least 5 minutes. A pressure air gun (filtered clean compressed air) was used to dry the surface of the endoscope and each pipeline, and the outer surface of the endoscope components and accessories was dried with sterile gauze. The dedicated endoscope cleaning button and perfusion connection tube were disassembled.

2.2.7. Sterilization. The dried endoscopes, buttons, water injection bottles, and other related accessories were sent to the central supply room for repackaging and packaging, and then sterilization with ethylene oxide gas (ethylene oxide sterilization machine from 3M: sterilization temperature, $55^{\circ} \mathrm{C}$; sterilization time, 1 hour; concentration, $\sim 600 \mathrm{mg}$; entire treatment time, 12 hours).

\subsection{Final Treatment of Inspection Room and Decontamination Room}

2.3.1. Diagnostic Equipment Such as Endoscope Host and Computer with Graphic System. Seventy-five percent alcohol was used to wipe and sterilize the surfaces of endoscope trolleys, endoscope host panels, endoscope display screens, computer display screens, computer mainframes, 
and computer mice for at least 30 minutes. The room was sterilized by ultraviolet radiation for 1 hour. After the final treatment at the bedside, the relevant equipment was pushed to the designated area for standby.

2.3.2. Inspection Room. The walls and floors were wiped with $2000 \mathrm{mg} / \mathrm{L}$ of chlorine-containing disinfectant. After more than 30 minutes, the room was sterilized with ultraviolet light for 1 hour.

2.3.3. Washing Room. The cleaning tank and full-tube perfusion device were continuously perfused with $2000 \mathrm{ppm}$ of effective chlorine solution for 30 minutes, followed by the addition of fresh water for 5 minutes and then drying. Next, $2000 \mathrm{mg} / \mathrm{L}$ of effective chlorine solution was used to wipe and disinfect from the reverse cleaning direction of the rinse tank. After 30 minutes, the surface was wiped with clean water. Finally, the tank was washed, and the drying table was wiped with disinfectant wipes. The disinfection room was closed and exposed to ultraviolet light for 1 hour, and the walls and floors were wiped with $2000 \mathrm{mg} / \mathrm{L}$ of chlorinecontaining disinfectant and allowed to stand for more than 30 minutes.

\subsection{Final Treatment and Detection of Nucleic Acid on the Surface of the Endoscope before the Withdrawal of Medical Staff and Equipment}

2.4.1. Nucleic Acid Detection and Medical Observation of Medical Staff. The medical staff, including doctors, nurses/technicians, and sterilizers, were subjected to the necessary 14-day isolation medical observations, and nucleic acid was tested twice in parallel with 24-hour intervals, and they returned to work only when the result is negative.

2.4.2. End-to-End Reprocessing before the Withdrawal of Endoscope-Related Equipment. After all the patients with COVID-19 were discharged from the hospital, the endoscope operation department and body, endoscope lumen, endoscope host, computer equipment, and treatment trolley were completely reprocessed (Table 1). Among them, the full-time engineer of the manufacturer mainly disassembled the endoscope host and the internal water/air pipe of the water pump/air pump and the air pump fan in the endoscope host were cleaned of the attached dust with a blower. After disassembling the endoscope, the cracks at the internal angles were cleaned and wiped with alcohol to prevent the entrapment of viruses. After cleaning all the equipment, fumigation treatment was carried out in a closed room with a hydrogen peroxide air sterilizer. Each surface of the above equipment was sampled twice to detect nucleic acids, and the results were all negative.

\section{Discussion}

The digestive tract is one of the target organs of SARS-CoV-2 [6]. Therefore, many studies have suggested that 3\%-65.9\% of patients with COVID-19 have gastrointestinal symptoms $[1,12]$. During the examination, aerosols were easily generated; therefore, the risk of infection of medical staff for endoscopy is extremely high.
TABLE 1: Nucleic acid sampling results before the evacuation of endoscopic diagnosis and treatment equipment for patients with COVID-19.

\begin{tabular}{|c|c|c|c|}
\hline & Sample & Times & Results \\
\hline \multirow[b]{2}{*}{ Endoscope } & Endoscope lumen & 2 & - \\
\hline & $\begin{array}{l}\text { Operation section+endoscope } \\
\text { body surface }\end{array}$ & 2 & - \\
\hline \multirow{2}{*}{ Trolley } & Trolley surface & 2 & - \\
\hline & Wheels & 2 & - \\
\hline \multirow{9}{*}{ Mainframe } & Display button & 2 & - \\
\hline & Display surface & 2 & - \\
\hline & Panel & 2 & - \\
\hline & Endoscopic Bayonet & 2 & - \\
\hline & Water and gas interface & 2 & - \\
\hline & $\begin{array}{l}\text { White balance calibration } \\
\text { device }\end{array}$ & 2 & - \\
\hline & Keyboard & 2 & - \\
\hline & Keyboard holder & 2 & - \\
\hline & Heat sink & 2 & - \\
\hline \multirow{5}{*}{$\begin{array}{l}\text { Computer of } \\
\text { graphic system }\end{array}$} & Button and panel & 2 & - \\
\hline & Heat sink & 2 & - \\
\hline & Data cable connection port & 2 & - \\
\hline & Display button & 2 & - \\
\hline & Display screen & 2 & - \\
\hline \multirow{3}{*}{$\begin{array}{l}\text { Mainframe } \\
\text { disassembly }\end{array}$} & $\begin{array}{l}\text { The inner water and gas pipe } \\
\text { of the main pump }\end{array}$ & 2 & - \\
\hline & Circuit board & 2 & - \\
\hline & Angle inside the mainframe & 2 & - \\
\hline
\end{tabular}

In response to this special situation, we propose the following: (1) epidemic emergency management: establish a specialty epidemic emergency team, comprising 5 senior doctors, 5 senior nurses, and 2 experienced disinfectors, to form a 12-member emergency team. (2) Team member management: to ensure the safety of patients and medical staff, the team members are given relevant protection and intensive training on the diagnosis process and multiple drills, including hand washing and putting on and taking off protective clothing, the endoscopy examination process, the endoscope reprocess, and other processes. All the staff must pass the evaluation before they can work, and the inspection personnel was relatively fixed to ensure proficiency in the diagnosis, treatment, and endoscope reprocessing. (3) Management of diagnostic equipment: as the only designated hospital in the municipal level, we prepared 2 endoscope mainframes, 12 endoscopes including gastrointestinal endoscopes, and 2 sets of endoscopy computer systems for the COVID-19 patients. The special endoscope host machine used by patients with COVID19 is a dedicated machine, and each patient used the same endoscope. After all the endoscopes have been used, they were decontaminated and sterilized according to the process. The department has temporarily sealed the equipment and confirmed that the nucleic acid test was negative before it can be used again. (4) Bedside 
preprocessing of the endoscope was one of the most critical steps. After the bedside examination, the endoscope was transfer to the reprocessing room needed a long time. If there was no preprocessing with multienzyme stock solution, during the transfer, the endoscope body and lumen could easily result in protein coagulation and crystallization, leading to the blockage of the endoscope lumen and retention of the virus, which can cause infection. (5) After the inspection, in addition to disinfecting the surface of the endoscope host and instrument of the graphic system, the internal water/air pipe and air pump of the water pump/air pump of the endoscope host were treated to avoid residual dust caused by the virus. After disassembly from the endoscope host, the circuit board of the host and gap between the angles in the host were cleaned and disinfected, and then fumigated with hydrogen peroxide air to ensure further virus kill. After this treatment, we sampled everywhere and tested twice for nucleic acids.

We performed detailed gastroscopy for 11 COVID-19 patients. In addition to zero-distance contact with patients, digestive tract secretions also directly contaminate the gastroscope, accessories, and work area. Due to the spreading characteristics of the virus, a series of disinfection measures were further taken, and strict implementation and inspection were carried out. No infections by medical staff occurred, and no viruses attached to the endoscope-related host and accessories, thereby reaching the standard of disinfection. We have accumulated experience in further effectively preventing the spread and infection of SARS$\mathrm{CoV}-2$.

\section{Conclusions}

The reprocessing of gastroenteroscopy in patients with SARS-CoV-2 infection was described. We found that bedside preprocessing with multienzyme solution is a key step. According to our procedures, the nucleic acid tests of endoscopists, decontamination personnel, endoscopes, and working platforms were all negative. Whether our experience is entirely applicable to the reprocessing of gastrointestinal endoscopy in patients with SARS-CoV-2 infection must be further explored.

\section{Data Availability}

The research data are available on request after institutional review board approval by contacting the corresponding author.

\section{Conflicts of Interest}

The authors declare that there is no conflict of interest regarding the publication of this paper.

\section{Authors' Contributions}

Xianqi Lin, Zhenyi Zhang, and Minzhao Gao have contributed equally to the manuscript.

\section{Acknowledgments}

This work was supported by the Task-Force Project on the Prevention and Control of Novel Coronavirus of Guangdong Province (20201113), the Three Major Constructions of Sun Yat-sen University (the Task-Force Project on the Prevention and Control of Novel Coronavirus of Sun Yat-sen University), and the Emergency Task-Force Project on the Prevention and Control of Novel Coronavirus of Zhuhai 2020.

\section{References}

[1] C. Huang, Y. Wang, X. Li et al., "Clinical features of patients infected with 2019 novel coronavirus in Wuhan, China," Lancet, vol. 395, no. 10223, pp. 497-506, 2020.

[2] N. Chen, M. Zhou, X. Dong et al., "Epidemiological and clinical characteristics of 99 cases of 2019 novel coronavirus pneumonia in Wuhan, China: a descriptive study," Lancet, vol. 395, no. 10223, pp. 507-513, 2020.

[3] T. Chen, H. C. Di Wu, W. Yan et al., "Clinical characteristics of 113 deceased patients with coronavirus disease 2019: retrospective study,” BMJ, vol. 368, p. m1091, 2020.

[4] Y. Song, P. Liu, X. L. Shi et al., "SARS-CoV-2 induced diarrhoea as onset symptom in patient with COVID-19," Gut, vol. 69, no. 6, pp. 1143-1144, 2020.

[5] L. Lin, X. Jiang, Z. Zhang et al., "Gastrointestinal symptoms of 95 cases with SARS-CoV-2 infection," Gut, vol. 69, no. 6, pp. 997-1001, 2020.

[6] F. Xiao, M. Tang, X. Zheng, Y. Liu, X. Li, and H. Shan, "Evidence for gastrointestinal infection of SARS-CoV-2," Gastroenterology, vol. 158, no. 6, pp. 1831-1833.e3, 2020.

[7] Y. Wu, C. Guo, L. Tang et al., "Prolonged presence of SARS-CoV-2 viral RNA in faecal samples," The Lancet Gastroenterology \& Hepatology, vol. 5, no. 5, pp. 434-435, 2020.

[8] W. Wang, Y. Xu, R. Gao et al., "Detection of SARS-CoV-2 in different types of clinical specimens," Journal of the American Medical Association, vol. 323, no. 18, pp. 1843-1844, 2020.

[9] National Standard of the People's Republic of China (Chinese), "Regulation for prevention and control of healthcare associated infection of airborne transmission disease in healthcare facilities WS/T511-2016," Chinese Journal of Infection Control, vol. 16, pp. 490-492, 2017.

[10] A. Repici, R. Maselli, M. Colombo et al., "Coronavirus (COVID-19) outbreak: what the department of endoscopy should know," Gastrointestinal Endoscopy, vol. 92, no. 1, pp. 192-197, 2020.

[11] National Standard of the People's Republic of China (Chinese), "Regulation for cleaning and disinfection technique of flexible endoscope WS507-2016," Chinese Journal of Infection Control, vol. 16, pp. 587-592, 2017.

[12] D. Wang, B. Hu, C. Hu et al., "Clinical characteristics of 138 hospitalized patients with 2019 novel coronavirus-infected pneumonia in Wuhan, China," JAMA, vol. 323, no. 11, pp. 1061-1069, 2020. 